\title{
Pregnant during the COVID-19 pandemic: Knowledge, concerns, attitudes and practices of Pakistani women
}

\author{
Rubina Izhar ${ }^{1,2,3}$, Samia Husain ${ }^{1,2}$, Muhammad A. Tahir ${ }^{1}$, Sonia Husain ${ }^{1,4}$, Saba Hussain ${ }^{5}$
}

\begin{abstract}
INTRODUCTION Data regarding pregnancy and related outcomes with COVID-19 are inconsistent, which leads to difficulties in counselling pregnant women. This brings uncertainty to pregnant women regarding mode of birth, transmission and issues that may occur in case they contract the disease. We conducted this study to assess the knowledge about COVID-19 risk during pregnancy and childbirth and to assess the concerns, attitudes, and practices of pregnant women during the pandemic.
\end{abstract}

METHODS A cross-sectional survey was carried out among 376 consenting pregnant women attending antenatal clinics in Karachi, Pakistan, between 1 July and 16 July 2020, using pretested questionnaires.

RESULTS A total of 376 pregnant women participated in the survey. Participants had inadequate knowledge about COVID-19 risk during pregnancy, including potential vertical transmission (58.5\%), preferred route of delivery (52\%), safety of breastfeeding (50\%), birth defects (44.7\%), rooming in and skin-to-skin contact (58.5\%). The majority (85.1\%) had a high concern score, and negative attitude (62.8\%). Only $43.6 \%$ said that they would not hide their symptoms, while $37.2 \%$ stated that they will deliver at hospital if they tested positive. Only $30.9 \%$ of respondents had good practices. During visits, $39.4 \%$ maintained social distancing and sanitized hands while only $37.2 \%$ said that they did not bring more than one person with them.

conclusions The study population had inadequate knowledge, negative attitude, and poor practices regarding pregnancy during the COVID-19 pandemic. More than half of the respondents thought that concealing symptoms and delivering at home would be better. Awareness programs are urgently needed.

\section{AFFILIATION \\ 1 Department of Obstetrics and Gynecology, Aziz Medical Center, Karachi, Pakistan \\ 2 Department of Obstetrics and Gynecology, Karachi Medical and Dental College, Karachi, Pakistan 3 Abbasi Shaheed Hospital, Karachi, Pakistan \\ 4 Department of Obstetrics and Gynecology, Aga Khan Hospital for Women Karimabad, Karachi, \\ Pakistan \\ 5 Department of Obstetrics and Gynecology, Dow Medical College, Dow University of Health Sciences, Karachi, Pakistan}

\section{CORRESPONDENGE TO}

Samia Husain. Department of Gynecology and Obstetrics, Aziz Medical Center, Nazimabad 3, Sindh 74600, Karachi, Pakistan. E-mail: samiahusain_scorpio@ hotmail.com

\section{KEYWORDS}

COVID-19, pregnancy, knowledge, attitudes, practices, Pakistan

Received: 25 December 2020

Revised: 1 February 2021

Accepted: 2 October 2021

\section{INTRODUCTION}

Infection from the coronavirus SARS-CoV-2 (COVID-19) was declared a pandemic on 11 March $2020^{1}$. Since then it has spread to 213 countries. Pakistan has been severely affected, with over 261917 cases and over 5522 deaths ${ }^{2}$. COVID-19 is a novel disease, therefore data on its the clinical course are sparse. Furthermore, its effects on pregnancy are not well known ${ }^{3}$.

Women generally are more likely to display higher levels of psychological distress after natural calamities ${ }^{4}$. Studies have shown high levels of anxiety and fear in pregnant women due to COVID-195. Pregnant women have started showing a greater amount of concern about COVID-196. They miss appointments and frequently show concern about transmission to their baby?.

Data regarding pregnancy outcomes are inconsistent, which leads to difficulties in counselling pregnant women. This brings uncertainty to pregnant women regarding mode of birth, transmission, and issues that may occur with breastfeeding, in case they contract the disease ${ }^{7}$. 
Although reports have been published on the knowledge, attitudes and practices of the general populations regarding COVID-19, data on pregnant women's perception of the effects of COVID-19 on pregnancy remain scarce ${ }^{8}$.

Counselling plays a major role in allaying fears and helps women cope with pregnancy-associated concerns. Proper counselling cannot be instituted if the providers are unaware of the concerns and current knowledge of their patient. We undertook this study to assess the pregnant women's knowledge about pregnancy in the pandemic and their attitude, concerns and practices regarding COVID-199.

\section{METHODS}

\section{Study design}

The present study is a multi-facility cross sectional survey conducted from 1 July 2020 to 16 July 2020 on pregnant women attending outpatient clinics of a public sector hospital, a private maternity home, and a private practice.

\section{Study settings}

The study was carried out in the outpatient clinics of obstetrics and gynecology of three hospitals in Karachi. Karachi is the biggest city of Pakistan and has a population of 20 million $^{10}$. Abbasi Shaheed hospital is the third largest tertiary care public sector hospital, Aziz medical center is a private secondary care facility that receives patients irrespective of socioeconomic status while the private practice is located in an area of the city which is frequented by the privileged of the city. The delivery rate for Abbasi Shaheed Hospital is 3600 per year, and around 100 women come for antenatal care each day. The antenatal clinic is held from 9 a.m. to 1 p.m., Monday through Saturday. These clinics are run by consultant obstetricians with their teams of resident doctors and are assisted by nurses. Aziz medical center has a delivery rate of 1000 per year and 40 women attend for antenatal care during each clinic. The clinic is run by two consultants who are assisted by office assistants and nurses. The clinic is held every Tuesday, Thursday and Sunday. The private practice has a delivery rate of 250 per year and 25 women attend for antenatal care in each clinic. The clinic is held twice a week. The clinic is run independently by one consultant obstetrician.

\section{Sample size}

We calculated the sample size using EpiCalc-2000. Our calculation was based on the following assumption: the proportion of good knowledge would be $50 \%$, level of confidence $95 \%$ and precision 5\%. The sample size was calculated to be 384 , and the sample size was increased by $10 \%$, to allow for losses.

\section{Study instrument}

Data were collected using a questionnaire. The questions were designed based on a literature search and frequently asked questions by women during counselling sessions. All questions were discussed with the expert committee that had two obstetricians, one pediatrician, one midwife, a translator, and a psychiatrist. Questions were written in
English then translated into Urdu. The questionnaire was then back translated into English. The questionnaire was checked for content and relevance by the authors. We pretested the instrument on 20 pregnant women who were not included in the final analysis. That pretesting showed that the internal consistency of the knowledge (Cronbach's alpha=0.888), concern (Cronbach's alpha=0.778), attitude (Cronbach's alpha $=0.678$ ) and practice (Cronbach's alpha=0.824) sections were good. Data were collected using a questionnaire which was divided into 4 sections: 1 ) section on demographic and pregnancy related characteristics; 2) knowledge section which had 10 questions covering risk of coronavirus in pregnancy, mode of delivery, breastfeeding and effects on baby, with responses of 'yes', 'no', or 'don't know'. A correct response was given 2 points and incorrect response or 'don't know' received 0 points; 3) attitude section had 5 questions that assessed attitude to healthcare in times of COVID-19 (five items), with response to each attitude question was recorded as either 'yes' (2 points), 'maybe' (1 point) and 'no' (0 points); 4) concern section had 5 questions that assessed concerns regarding pregnancy during COVID-19 pandemic, with response to each concern item was recorded as follows: 'yes' (2 points) and 'no' (1 point); and 5) practice section had 6 questions addressing the woman's practices during pregnancy, with each question having two responses, 'yes' (1 point) and 'no' (0 points).

\section{Study population}

Pregnant women, with no symptoms of coronavirus were approached for the survey by the authors. Nonprobability consecutive sampling technique was used. Women attending an outpatient department due to suspected or diagnosed labor and women with obstetric emergencies were excluded. We also excluded women with known psychiatric disorders. The women were approached before their antenatal visit in the waiting area. Women were interviewed by the authors in the clinics of obstetrics and gynecology who were trained to administer the questionnaire. Both the participant and the interviewer wore facemasks during the interview. Translation into native languages was done in cases where the respondents had lower levels of education.

\section{Data collection and statistical analysis}

After obtaining informed consent, we briefed each participant about the purpose of study. Anonymity was ensured and no particulars that could identify the participant were collected. After the data collection, each woman was given accurate and up-to-date information on all aspects of pregnancy and COVID-19. They were encouraged to ask questions and all their misconceptions were cleared. They were also provided the contact numbers of the authors and information leaflets.

Knowledge score was calculated for the 10 knowledge questions as: $1-12$ inadequate and $\geq 13$ adequate. Attitude score was: $\leq 6$ negative and $\geq 7$ positive. Level of concern score was: $\leq 6$ low level and $\geq 7$ high.

Practice score was also calculated for practices regarding pregnancy: $1-4$ poor practices and $\geq 5$ good. 
Statistical Package for Social Science (SPSS) software version 23 was used to analyze data. Descriptive statistical analysis was used to describe items included in the survey. Numbers and mean percentages were used to describe the categorical data. Odds ratios (ORs) with 95\% confidence intervals (Cls) were calculated for variables of interest. A $\mathrm{p}<0.05$ was considered statistically significant.

\section{RESULTS}

We approached 466 women to participate and 37 refused participation. Therefore, 429 were included. Of these 429, we excluded 43 due to missing responses; the response rate was $90 \%$. Only those questionnaires that had no missing responses were included in the final analysis. More than two-thirds $(n=264 ; 70 \%)$ were aged $<30$ years and were in their second (39.4\%) or third (39.4\%) trimester of pregnancy. Table 1 summarizes sociodemographic and pregnancy related characteristics of the study population and scores obtained during the survey.

Only $39.4 \%$ women had adequate knowledge. The knowledge was inadequate regarding vertical transmission to baby (58.5\%), mode of delivery (52\%), breastfeeding (50\%), birth defects (44.7\%), rooming in and skin-toskin contact (58.5\%). Table 2 summarizes the knowledge section.

The majority (85.1\%) had a high concern score and negative attitude score $62.8 \%$. Less than one-third (30.9\%) of the respondents had good practices. Table 3 summarizes

Table 1. Sociodemographic, pregnancy characteristics of study population and scores obtained during the survey $(\mathrm{N}=376)$

\begin{tabular}{|lc}
\hline Characteristics & $\boldsymbol{n}(\%)$ \\
\hline Age (years) & \\
\hline $19-24$ & $144(38.3)$ \\
\hline $25-29$ & $120(31.9)$ \\
\hline $30-34$ & $64(17.0)$ \\
\hline $35-39$ & $28(7.4)$ \\
$\geq 40$ & $20(5.3)$ \\
\hline
\end{tabular}

Education level

Graduate

$80(21.3)$

Inter $\quad 88$ (23.4)

Matric

$108(28.7)$

Not formally educated

$100(26.6)$

Monthly income (PKR)*

$\begin{array}{lr}15000-50000 & 168(44.7) \\ >50000 & 120(31.9) \\ <15000 & 88(23.4)\end{array}$

\section{Employment status}

Employed

$140(37.2)$

Unemployed

$236(62.8)$
Table 1. Continued

\begin{tabular}{lr}
\hline Characteristics & $\boldsymbol{n}(\%)$ \\
\hline Residential area & $108(28.7)$ \\
\hline Rural & $268(71.3)$ \\
\hline Urban & \\
\hline Source of information on COVID-19 & $81(21.5)$ \\
\hline Doctors/nurses/midwives & $106(28.2)$ \\
\hline Social media & $102(27.1)$ \\
\hline Newspaper/news channels & $87(23.1)$ \\
\hline Websites (WHO, CDC, Pakistani)
\end{tabular}

\section{Place of induction of participant into the study}

Public sector hospital $128(34)$

Private secondary care $162(43.1)$

Private practice 86 (22.9)

Gestational age (weeks)

$<13$ $80(21.3)$

$13-26$ 148 (39.4)

$>26$ $148(39.4)$

Parity

0 51 (13.6)

1 145 (38.6)

2 108 (28.7)

3 40 (10.6)

$>3$ 32 (8.5)

Scores, mean $\pm S D$

Knowledge

$11.29 \pm 6.86$

Concern

$8.30 \pm 1.482$

Attitude

$5.56 \pm 3.433$

Practices

$3.04 \pm 2.13$

Knowledge

Adequate

$148(39.4)$

Inadequate

\section{Concern}

High

Low

\section{Attitude}

Negative

$236(62.8)$

Positive

$140(37.2)$

\section{Practices}

Good

Poor

$260(69.1)$

SD: standard deviation. *PKR: 1 US\$ about 160 Pakistani rupees. Knowledge score for the 10 knowledge questions was: $1-12$ inadequate and $\geq 13$ adequate. Attitude score was: $\leq 6$ negative and $\geq 7$ positive. Concern score was: $\leq 6$ low level and $\geq 7$ high level. Practices during pregnancy score: $1-4$ poor and $\geq 5$ good. 
attitudes, concerns and practices of the population.

Table 4 summarizes predictors of knowledge and good practices and Table 5 summarizes predictors of high concern and positive attitudes. Factors associated with adequate knowledge were: age 30-34 years $(A O R=0.17$; 95\% Cl: 0.06-0.50, $\mathrm{p}<0.001)$; graduate level education $(A O R=6.05 ; 95 \% \mathrm{Cl}: 2.27-16.12, p=0.001)$; rural residence (AOR=0.12; 95\% Cl: 0.05-0.31, p<0.001); and having

\section{Table 2. Knowledge of COVID-19 among pregnant women}

\begin{tabular}{|c|c|c|c|}
\hline \multirow[t]{2}{*}{ Knowledge of COVID-19 and pregnancy } & Yes* & Don't know & No \\
\hline & $n(\%)$ & $n(\%)$ & $n(\%)$ \\
\hline K1. Pregnant women are more susceptible to corona virus & $284(75.5)$ & $8(2.1)$ & $84(22.3)$ \\
\hline K2. If I contract coronavirus after 28 weeks, I may have more complications & $268(71.3)$ & $16(4.3)$ & $92(24.5)$ \\
\hline $\begin{array}{l}\text { K3. There is a slight chance of giving coronavirus to my baby if I have coronavirus during } \\
\text { pregnancy or childbirth }\end{array}$ & $220(58.5)$ & $12(3.2)$ & $144(38.3)$ \\
\hline K4. If I get coronavirus, I can still deliver normally & $196(52.1)$ & $28(7.4)$ & $152(40.4)$ \\
\hline K5. I should breastfeed even if I have coronavirus & $188(50.0)$ & $20(5.3)$ & $168(44.7)$ \\
\hline K6. During breastfeeding my face should be covered in case I get the coronavirus & $172(45.7)$ & $44(11.7)$ & $160(42.6)$ \\
\hline K7. If I get coronavirus, I may still touch my baby (skin-to-skin contact) & $220(58.5)$ & $16(4.3)$ & $140(37.2)$ \\
\hline K8. Coronavirus during pregnancy can cause births earlier than the due date & $188(50.0)$ & $24(6.4)$ & $164(43.6)$ \\
\hline K9. According to current evidence coronavirus does not lead to birth defects & $168(44.7)$ & $32(8.5)$ & $176(46.8)$ \\
\hline K10. If I get coronavirus I can still be in the same room as my baby (rooming in) & $220(58.5)$ & $16(4.3)$ & $140(37.2)$ \\
\hline
\end{tabular}

*Correct responses, correct response rate (women who answered all questions correctly) 51.98\%.

Table 3. Concerns, attitudes, and preventive practices during pregnancy, in the pandemic

\begin{tabular}{|c|c|c|c|}
\hline \multirow[t]{2}{*}{ Concerns regarding COVID-19 and pregnancy } & Yes & No & \\
\hline & $n(\%)$ & $n(\%)$ & \\
\hline C1. Are you concerned about the coronavirus outbreak? & $352(93.6)$ & $24(6.4)$ & \\
\hline C2. Do you feel more vulnerable/weak during the outbreak because you are pregnant? & $208(55.3)$ & $168(44.7)$ & \\
\hline C3. Do you constantly keep thinking that you may have or already have coronavirus? & $192(51.1)$ & $184(48.9)$ & \\
\hline C4. Do you think that your baby might get infected after it's born? & $256(68.1)$ & $120(31.9)$ & \\
\hline C5. Do you think that you might get infected or right after delivery? & $236(62.8)$ & $140(37.2)$ & \\
\hline \multirow[t]{2}{*}{ Attitudes } & Yes & No & Maybe \\
\hline & $n(\%)$ & $n(\%)$ & $n(\%)$ \\
\hline Al. I trust the information provided by my doctor & $272(72.3)$ & $100(26.6)$ & $4(1.1)$ \\
\hline $\begin{array}{l}\text { A2. I am sure I will be looked after in the best possible way in case I test positive for } \\
\text { coronavirus }\end{array}$ & $188(50.0)$ & $180(47.9)$ & $8(2.1)$ \\
\hline A3. I feel supported by my obstetrics team during visits & $260(69.1)$ & $116(30.9)$ & $\mathrm{O}(0.0)$ \\
\hline A4. I will not conceal my symptoms from my doctor in case I am infected & $164(43.6)$ & $200(53.2)$ & $12(3.2)$ \\
\hline A5. I will deliver at hospital if I become COVID-19 positive & $140(37.2)$ & $216(57.4)$ & $20(5.3)$ \\
\hline \multirow[t]{2}{*}{ Practices during pregnancy } & Yes & No & \\
\hline & $n(\%)$ & $n(\%)$ & \\
\hline P1. I did not skip any appointments due to coronavirus & $224(59.6)$ & $152(40.4)$ & \\
\hline P2. I take precautions whenever I leave my house (cover my face) & $200(53.2)$ & $176(46.8)$ & \\
\hline P3. I used telehealth during this pandemic & $180(47.9)$ & $196(52.1)$ & \\
\hline P4. I have a 30-day supply of medicines and supplements & $256(68.1)$ & $120(31.9)$ & \\
\hline P5. I maintained social distancing during visits and sanitized hands & $148(39.4)$ & $228(60.6)$ & \\
\hline P6. I did not bring more than one person to my visits & $136(36.2)$ & $240(63.8)$ & \\
\hline
\end{tabular}


monthly household income $>50000$ PKR (AOR=0.017; 95\% Cl: 0.05-0.58, p=0.004).

Factors associated with good practices were: age 25-29 years (AOR=0.11; 95\% Cl: 0.03-0.28, $\mathrm{p}<0.001$ ); intermediate education $(\mathrm{AOR}=0.18 ; 95 \% \mathrm{Cl}: 0.06-0.54$, $\mathrm{p}=0.002)$; and having monthly income $>50000$ PKR (AOR=12.23; 95\% Cl: 2.80-53.28, $p=0.001$ ).

Factors associated with high concerns were: age 19-24

Table 4. Sociodemographic predictors of adequate knowledge and good practices regarding COVID-19 in pregnant women

\begin{tabular}{|c|c|c|c|c|c|c|c|c|}
\hline & \multicolumn{4}{|c|}{ Knowledge scores } & \multicolumn{4}{|c|}{ Practice scores } \\
\hline & $\begin{array}{c}\text { Adequate } \\
(n=148)\end{array}$ & $\begin{array}{c}\text { Inadequate } \\
(n=228)\end{array}$ & $\operatorname{AOR}(95 \% \mathrm{CI})^{a}$ & $p$ & $\begin{array}{c}\text { Good } \\
(n=116)\end{array}$ & $\begin{array}{c}\text { Poor } \\
(n=260)\end{array}$ & AOR $(95 \% C I)^{a}$ & $p$ \\
\hline \multicolumn{9}{|l|}{ Age (years) } \\
\hline $19-24$ & 60 & 84 & $0.348(0.13-0.93)$ & $0.036^{*}$ & 48 & 96 & $0.409(0.15-1.05)$ & 0.064 \\
\hline $25-29$ & 40 & 80 & $0.20(0.07-0.54)$ & $0.002^{*}$ & 16 & 104 & $0.11(0.03-0.28)$ & $0.001^{*}$ \\
\hline $30-34$ & 20 & 44 & $0.17(0.06-0.50)$ & $0.001^{*}$ & 24 & 40 & $0.52(0.19-1.42)$ & 0.203 \\
\hline$\geq 35$ (Ref.) & 28 & 20 & 1 & & 28 & 20 & 1 & \\
\hline \multicolumn{9}{|l|}{ Education level } \\
\hline Graduate & 56 & 24 & $6.05(2.27-16.12)$ & $0.001^{*}$ & 52 & 28 & $1.46(0.52-4.10)$ & 0.473 \\
\hline Intermediate & 24 & 64 & $0.69(0.27-1.70)$ & 0.420 & 16 & 72 & $0.18(0.06-0.54)$ & $0.002^{*}$ \\
\hline Matric & 48 & 60 & $1.55(0.66-3.63)$ & 0.303 & 32 & 76 & 0.79 (0.30-2.09) & 0.646 \\
\hline Not formally educated (Ref.) & 20 & 80 & 1 & & 16 & 84 & 1 & \\
\hline \multicolumn{9}{|l|}{ Monthly income $(P K R)^{b}$} \\
\hline $15000-50000$ & 72 & 96 & $0.62(0.23-1.63)$ & 0.334 & 44 & 124 & $7.84(1.85-33.08)$ & $0.005^{*}$ \\
\hline$>50000$ & 60 & 60 & $0.175(0.05-0.58)$ & $0.004^{*}$ & 68 & 52 & $12.23(2.80-53.28)$ & $0.001^{*}$ \\
\hline$<15000$ (Ref.) & 16 & 72 & 1 & & 4 & 84 & 1 & \\
\hline \multicolumn{9}{|l|}{ Employment status } \\
\hline Employed & 80 & 60 & $1.93(0.94-3.94)$ & 0.072 & 76 & 64 & $1.91(0.89-4.12)$ & 0.096 \\
\hline \multicolumn{9}{|l|}{ Unemployed } \\
\hline (Ref.) & 68 & 168 & 1 & & 40 & 196 & 1 & \\
\hline \multicolumn{9}{|l|}{ Residential area } \\
\hline Rural & 12 & 96 & $0.12(0.05-0.31)$ & $0.001^{*}$ & 12 & 96 & $0.76(0.29-1.97)$ & 0.583 \\
\hline Urban (Ref.) & 136 & 132 & 1 & & 104 & 164 & 1 & \\
\hline
\end{tabular}

AOR: adjusted odds ratio. aVariables that showed significant associations on bivariate analysis were used in multivariate logistic regression to identify the independent predictors. *Statistically significant on multivariate analysis. bPKR: 1 US $\$$ about 160 Pakistani rupees.

Table 5. Sociodemographic predictors of high concerns and positive attitudes of women regarding pregnancy in COVID-19 era

\begin{tabular}{|c|c|c|c|c|c|c|c|c|}
\hline & \multicolumn{4}{|c|}{ Concern scores } & \multicolumn{4}{|c|}{ Attitude scores } \\
\hline & $\begin{array}{c}\text { High } \\
(n=320)\end{array}$ & $\begin{array}{c}\text { Low } \\
(n=56)\end{array}$ & $\operatorname{AOR}(95 \% C I)^{a}$ & $p$ & $\begin{array}{c}\text { Negative } \\
(n=236)\end{array}$ & $\begin{array}{l}\text { Positive } \\
(n=140)\end{array}$ & $\operatorname{AOR}(95 \% C I)^{a}$ & $p$ \\
\hline \multicolumn{9}{|c|}{ Age (years) } \\
\hline $19-24$ & 132 & 12 & $8.01(2.05-31.22)$ & $0.003^{*}$ & 88 & 56 & $0.42(0.16-1.11)$ & 0.082 \\
\hline $25-29$ & 104 & 16 & $4.49(1.24-16.23)$ & $0.022^{*}$ & 88 & 32 & $0.22(0.08-0.58)$ & $0.002^{*}$ \\
\hline $30-34$ & 48 & 16 & $0.98(0.29-3.31)$ & 0.977 & 48 & 16 & $0.107(0.03-0.30)$ & $0.001^{*}$ \\
\hline$\geq 35$ (Ref.) & 36 & 12 & 1 & & 12 & 36 & 1 & \\
\hline
\end{tabular}


Table 5. Continued

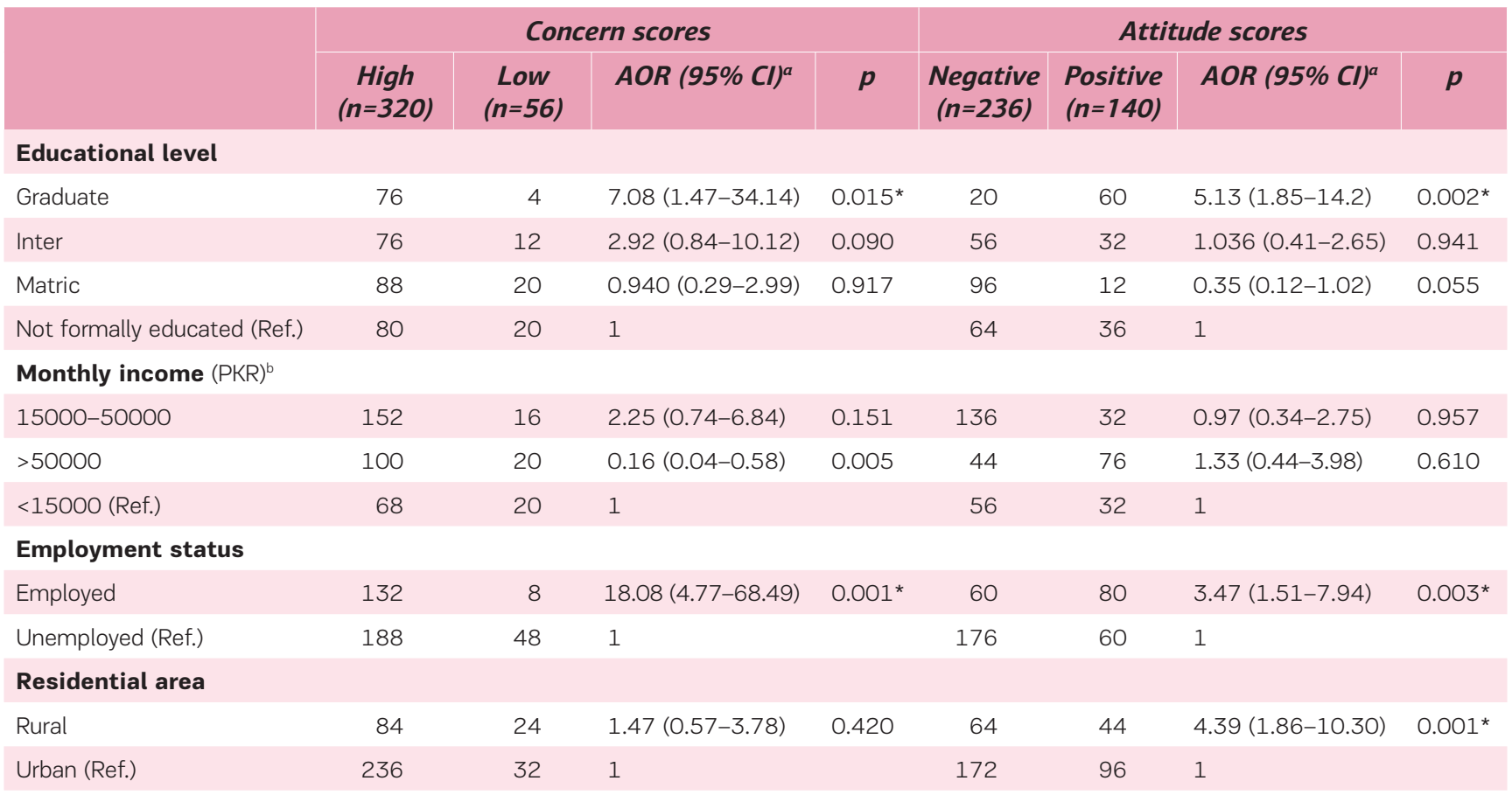

AOR: adjusted odds ratio. a Variables that showed significant associations on bivariate analysis were used in multivariate logistic regression to identify the independent predictors. *Statistically significant on multivariate analysis. b PKR: 1 US\$ about 160 Pakistani rupees.

years (AOR=8.01; 95\% Cl: 2.05-31.22, $p=0.003)$; graduate (AOR=7.08; 95\% Cl: 1.47-34.14, $\mathrm{p}=0.015)$; being employed $(A O R=18.08 ; 95 \% \mathrm{Cl}: 4.77-68.49, p=0.001)$; and having monthly income $>50000$ PKR (AOR=0.16; 95\% Cl: 0.040.58, $p=0.005$ ).

Factors associated with positive attitudes were: age 30-34 years $(\mathrm{AOR}=0.17 ; 95 \% \mathrm{Cl}$ : 0.06-0.50, $\mathrm{p}<0.001)$; being employed (AOR=3.47; 95\% Cl: 1.51-7.94, $\mathrm{p}=0.003$ ); graduate $(A O R=5.13 ; 95 \% \mathrm{Cl}: 1.85-14.2, \mathrm{p}=0.002)$; and rural residence $(A O R=4.39 ; 95 \% \mathrm{Cl}: 1.86-10.30, \mathrm{p}<0.001)$.

\section{DISCussion}

Knowledge about coronavirus and how it affects pregnancy is of paramount importance because it helps mold positive attitudes, allays fears and concerns and leads to good preventive practices in pregnancy. Our cross-sectional survey shows that participating Pakistani pregnant women had inadequate knowledge about the relationship between pregnancy and COVID-19. Our findings are in agreement with a Turkish study ${ }^{9}$ on pregnant women that also showed restricted knowledge but are in contrast with the Chinese study ${ }^{11}$ where three-quarters of the women had adequate knowledge. However, in that study women gained their information from doctors. Our women gathered information from social media (28.2\%) and therefore had lower levels of accurate knowledge. Previous studies on knowledge of COVID-19 specifically were largely promising in general populations $^{12}$ as well as pregnant population ${ }^{13}$ but the same is not true for knowledge about COVID-19 and its effects on pregnancy.
Knowledge on the subject of vertical transmission has evolved tremendously, earlier reports from China were hugely favorable but later reports depicted a theoretical risk and increased prevalence of preterm deliveries was also noticed ${ }^{14,15}$. Most of the respondents knew that pregnancy can make one more susceptible to COVID-19; but the chance of transmitting it to their newborn during childbirth is, at best, slight. It was encouraging to see that women were aware of new updates. Guidance on breastfeeding, rooming in and skin-to-skin contact differs slightly. WHO recommends breastfeeding for all women, if they are fit to do so $^{16}$. The American Academy of Pediatricians ${ }^{17}$ recommends expressed breast milk and Royal College of Obstetricians and Gynecologists also advises against direct breast feeding in cases of severe infection ${ }^{18}$. Our study participants did not have adequate knowledge of breastfeeding and newborn care. Older age, higher education and monthly income and urban residence were predictors of adequate knowledge in our study. COVID-19 is a public health problem, therefore counselling sessions in outpatient and delivery suites should be started to help dispel misconceptions.

Due to lack of concrete and convincing evidence for vertical transmission, women can have vaginal delivery even if they have COVID-19 ${ }^{19}$. Knowledge regarding mode of delivery was also inadequate. In our study, only 52\% of women thought that expecting mothers who test positive could still have a vaginal delivery. More than half $(57.4 \%)$ of the women preferred giving birth at home in case they tested positive for COVID-19. Asymptomatic COVID-19 patients have been shown to develop an exacerbation of 
pneumonia, postpartum. This can prove fatal and lead to an increased morbidity and mortality ${ }^{20}$. Moreover, obstetric emergencies that occur out of hospital and the delays that maybe encountered during transportation may aggravate maternal morbidity and mortality. Delivering outside hospital can therefore be extremely dangerous and directive counselling is necessary in this regard ${ }^{21}$.

Fear of contracting coronavirus during, or just after delivery, is also a huge area of concern. In our study around two-thirds (62.8\%) of women thought that they would get infected during or after birth, compared to 35\% of women in a Turkish study ${ }^{9}$. Furthermore, 68.1\% thought that their baby might get infected after being born. These women need to be informed that fetomaternal outcomes are superior to births out of hospital. If proper infection control and prevention strategies are employed, the chances of having an infection are lower than those of delivering at home where the birth attendant may or may not use a mask and deliver the baby with proper aseptic technique ${ }^{21}$.

Furthermore, only one-third of expecting mothers (38.1\%) had a positive attitude, which is in stark contrast to the Turkish study $(85 \%)^{9}$ and Chinese study $(90 \%)^{11}$. It was alarming that more than half $(53.2 \%)$ of the women stated they would hide their symptoms from their attending maternity care provider. This negative attitude can lead to exponential damage in settings where one pregnant woman comes in contact with many caregivers. Unexpected exposure from seemingly asymptomatic women has led to outbreaks in New York. In Pakistan, unlike New York, universal screening has not been implemented due to lack of resources ${ }^{22}$. Moreover, these women may decompensate post-delivery and multidisciplinary care maybe needed. Data from New York has shown that most (70\%) of the COVID-19 patients admitted to a labor ward were asymptomatic ${ }^{23}$. Another misconception therefore needs to be dispelled. Women with symptoms are actually at a higher risk and need to deliver at a health facility to ensure proper monitoring. In our study, older age, employment and higher education, were predictors of positive attitude. Therefore young, unemployed women with lower academic achievement are sensitive to the prevalent infodemic and should not be neglected. They need a reliable source of information so that myths are busted and positive attitudes become more prevalent.

Nine out of every ten (93.65\%) women in our study were concerned about the outbreak and more than half (55\%) felt weak because of pregnancy, during this pandemic. These numbers are similar to those reported from Turkey, where $80 \%$ women felt concerned and $52 \%$ felt vulnerable ${ }^{9}$. A recent analysis from Italy ${ }^{5}$ showed that negative emotional constructs were more common after the pandemic was declared. Psychological support is therefore crucial for the pregnant population. A recent analysis has reported high psychological distress and anxiety in pregnant women during COVID-1924.

Four out of every ten (40.4\%) women skipped appointments due to the coronavirus; the attendance at our clinics fell during the pandemic, which is similar to the trend in China ${ }^{7}$. Telehealth services were also used during the pandemic in many countries including Pakistan ${ }^{25}$. Around half of our women (47.9\%) used some form of telehealth during their pregnancy. The preventive practices during antenatal visits were alarming; around two-thirds neither sanitized their hands nor maintained social distancing during their antenatal visits. Pakistan is a densely populated country and social distancing may not be practical in outpatient areas. Recently WHO has urged the use of facemasks in these settings ${ }^{26}$, but our women did not cover their faces when leaving for places where they anticipated crowds. Pakistan has a high maternal mortality ratio (142.5 per 100000 live births) and infection in this susceptible population can be reduced if preventive measures are adopted ${ }^{27}$. In our study women with high household income and higher education had good practices. Preventive measures for COVID-19 are simple; campaigns that stress the importance of social distancing and mask use should be commenced in outpatient clinics.

We recommend that the following steps should be taken to combat the infodemic. The government should start media campaigns to disseminate updated information on COVID-19 and pregnancy. Hospital administrations should paste an infographic on safety of childbirth in hospitals and breastfeeding newborns during pandemics. Each pregnant women should receive at least one counselling session during her visits that addresses her concerns on COVID-19 from a midwife, if needed a psychiatric assessment should be done. Finally, at the time of admission to the labor suite each pregnant woman should be counseled on all aspects of breastfeeding and newborn care during the pandemic.

\section{Strengths and limitations}

To our knowledge this is the first study from Pakistan that reports findings on knowledge, attitudes, concerns and practices of pregnant women about pregnancy during the pandemic. It has a large sample size and is a multi-facility design to ensure generalizability of results to the population.

Our study has one major limitation. It is a cross-sectional survey and only pregnant women in Karachi who attended outpatient clinics were included. In addition, we did not employ any measure to assess anxiety or fear. We excluded all women with a history of psychiatric disorder but high level of concern needs to be evaluated further. Despite these limitations it is a useful contribution to guide authorities about the looming threat of inappropriate healthcare utilization.

\section{CONCLUSIONS}

The study population had inadequate knowledge, negative attitude, and poor preventive practices regarding COVID-19. High quality counselling programs to disseminate accurate and up-to-date information are urgently needed.

\section{REFERENCES}

1. Cucinotta D, Vanelli M. WHO Declares COVID-19 a Pandemic. Acta Biomed. 2020;91(1):157-160. doi:10.23750/abm.v91i1.9397 
2. Coronavirus disease (COVID-19): Data as received by WHO from national authorities by 10:00 CEST, 4 July 2020. World Health Organization; 2020. Situation Report 166. Accessed August 2, 2020. https://www. who.int/docs/default-source/coronaviruse/situationreports/20200704-covid-19-sitrep-166.pdf

3. Rasmussen SA, Smulian JC, Lednicky JA, Wen TS, Jamieson DJ. Coronavirus Disease 2019 (COVID-19) and pregnancy: what obstetricians need to know. Am J Obstet Gynecol. 2020;222(5):415-426. doi:10.1016/j.ajog.2020.02.017

4. Tolin DF, Foa EB. Sex differences in trauma and posttraumatic stress disorder: a quantitative review of 25 years of research. Psychol Bull. 2006;132(6):959992. doi:10.1037/0033-2909.132.6.959

5. Ravaldi C, Wilson A, Ricca V, Homer C, Vannacci A. Pregnant women voice their concerns and birth expectations during the COVID-19 pandemic in Italy. Women Birth. 2021;34(4):335-343. doi:10.1016/j.wombi.2020.07.002

6. Rashidi Fakari F, Simbar M. Coronavirus Pandemic and Worries during Pregnancy; a Letter to Editor. Arch Acad Emerg Med. 2020;8(1):e21. Accessed February 1, 2021. https://www.ncbi.nlm.nih.gov/pmc/articles/ PMC7075675/pdf/aaem-8-e21.pdf

7. Gu XX, Chen K, Yu H, Liang GY, Chen H, Shen Y. How to prevent in-hospital COVID-19 infection and reassure women about the safety of pregnancy: Experience from an obstetric center in China. J Int Med Res. 2020;48(7):300060520939337. doi:10.1177/0300060520939337

8. Berthelot N, Lemieux R, Garon-Bissonnette J, DrouinMaziade C, Martel É, Maziade M. Uptrend in distress and psychiatric symptomatology in pregnant women during the coronavirus disease 2019 pandemic. Acta Obstet Gynecol Scand. 2020;99(7):848-855. doi:10.1111/aogs.13925

9. Yassa M, Birol P, Yirmibes C, et al. Near-term pregnant women's attitude toward, concern about and knowledge of the COVID-19 pandemic. J Matern Fetal Neonatal Med. 2020;33(22):3827-3834. doi:10.1080/14767058.2020.1763947

10. Shabbir W, Pilz J, Naeem A. A spatial-temporal study for the spread of dengue depending on climate factors in Pakistan (2006-2017). BMC Public Health. 2020;20(1):995. doi:10.1186/s12889-020-08846-8

11. Lee TY, Zhong Y, Zhou J, He X, Kong R, Ji J. The outbreak of coronavirus disease in China: Risk perceptions, knowledge, and information sources among prenatal and postnatal women. Women Birth. 2021;34(3):212218. doi:10.1016/j.wombi.2020.05.010

12. Zhong BL, Luo W, Li HM, et al. Knowledge, attitudes, and practices towards COVID-19 among Chinese residents during the rapid rise period of the COVID-19 outbreak: a quick online cross-sectional survey. Int J Biol Sci. 2020;16(10):1745-1752. doi:10.7150/ijbs.45221

13. Anikwe CC, Ogah CO, Anikwe IH, Okorochukwu BC, Ikeoha CC. Coronavirus disease 2019: Knowledge, attitude, and practice of pregnant women in a tertiary hospital in Abakaliki, southeast Nigeria. Int J Gynaecol Obstet. 2020;151(2):197-202. doi:10.1002/ijgo.13293

14. Levy A, Yagil Y, Bursztyn M, Barkalifa R, Scharf S, Yagil C. ACE2 expression and activity are enhanced during pregnancy. Am J Physiol Regul Integr Comp Physiol. 2008;295(6):R1953-R1961. doi:10.1152/ajpregu.90592.2008

15. Sentilhes L, De Marcillac F, Jouffrieau C, et al. Coronavirus disease 2019 in pregnancy was associated with maternal morbidity and preterm birth. Am J Obstet Gynecol. 2020;223(6):914.e1-914.e15. doi:10.1016/j.ajog.2020.06.022

16. Infographics on Pregnancy, Childbirth, breastfeeding and COVID-19. Pregnancy and childbirth during COVID-19. World Health Organization. Accessed July 20, 2020.

https://www.who.int/reproductivehealth/publications/ emergencies/Pregnancy-1-1200x1200.png

17. Coronavirus Disease (COVID-19) and Breastfeeding. Centers for Disease Control and Prevention. Updated March 25, 2021. Accessed February 1, 2021. https:// www.cdc.gov/breastfeeding/breastfeeding-specialcircumstances/maternal-or-infant-illnesses/covid-19and-breastfeeding.html

18. Coronavirus (COVID-19) Infection in Pregnancy: Information for healthcare professionals, Version 2. Royal College of Obstetricians and Gynaecologists; 2020. March 13, 2020. Accessed July 20, 2020. https://web.archive.org/web/20200315005540/ https://www.rcog.org.uk/globalassets/documents/ guidelines/coronavirus-covid-19-infection-inpregnancy-v2-20-03-13.pdf

19. Chen H, Guo J, Wang C, et al. Clinical characteristics and intrauterine vertical transmission potential of COVID-19 infection in nine pregnant women: a retrospective review of medical records. Lancet. 2020;395(10226):809815. doi:10.1016/S0140-6736(20)30360-3

20. Reis HLBD, Boldrini NAT, Caldas JVJ, Paz APCD, Ferrugini CLP, Miranda AE. Severe coronavirus infection in pregnancy: challenging cases report. Rev Inst Med Trop Sao Paulo. 2020;62:e49. doi:10.1590/s1678-9946202062049

21. Grünebaum A, McCullough LB, Bornstein E, Klein R, Dudenhausen JW, Chervenak FA. Professionally responsible counseling about birth location during the COVID-19 pandemic. J Perinat Med. 2020;48(5):450452. doi:10.1515/.jpm-2020-0183

22. Prabhu M, Cagino K, Matthews KC, et al. Pregnancy and postpartum outcomes in a universally tested population for SARS-CoV-2 in New York City: a prospective cohort study. BJOG. 2020;127(12):1548-1556. doi:10.1111/1471-0528.16403

23. Fassett MJ, Lurvey LD, Yasumura L, et al. Universal SARS-Cov-2 Screening in Women Admitted for Delivery in a Large Managed Care Organization. Am J Perinatol. 2020;37(11):1110-1114. doi:10.1055/s-0040-1714060

24. Taubman-Ben-Ari O, Chasson M, Abu Sharkia S, Weiss 
E. Distress and anxiety associated with COVID-19 among Jewish and Arab pregnant women in Israel. J Reprod Infant Psychol. 2020;38(3):340-348. doi:10.1080/02646838.2020.1786037

25. Ali NA, Khoja A. Telehealth: An Important Player During the COVID-19 Pandemic. Ochsner J. 2020;20(2):113114. doi:10.31486/toj.20.0039

26. Missoni E, Armocida B, Formenti B. Face Masks for All and All for Face Masks in the COVID-19 Pandemic: Community Level Production to Face the Global Shortage and Shorten the Epidemic. Disaster Med Public Health Prep. 2021;15(1):e29-e33. doi:10.1017/dmp.2020.207

27. Mubeen K, Jan R, Sheikh S, Lakhani A, Badar SJ. Maternal and newborn outcomes of care from community midwives in Pakistan: A retrospective analysis of routine maternity data. Midwifery. 2019;79:102553. doi:10.1016/j.midw.2019.102553

\section{CONFLICTS OF INTEREST}

The authors have completed and submitted the ICMJE Form for Disclosure of Potential Conflicts of Interest and none was reported.

\section{FUNDING}

There was no source of funding for this research.

ETHIGAL APPROVAL AND INFORMED CONSENT

This research was approved by the institutional ethics committee of Aziz Medical Center (IEC/AZIZ/1639/2020). Furthermore, all participating facilities provided permission to approach women during antenatal visits.

\section{DATA AVAILABILITY}

The data supporting this research are available from the authors on reasonable request.

\section{AUTHORS' CONTRIBUTIONS}

RI: conceptualization, methodology, supervision, visualization, project management, data curation. SaH: software, data curation, investigation, writing and original draft preparation, validation, formal analysis, writing, reviewing and editing. MAT: investigation, writing and original draft preparation, validation, formal analysis, writing, reviewing and editing. SoH: writing and original draft preparation, validation, formal analysis, writing, reviewing and editing. SabH: writing and original draft preparation, validation, formal analysis, writing, reviewing and editing.

\section{PROVENANGE AND PEER REVIEW}

Not commissioned; externally peer reviewed. 